\title{
A novel Lipid-Carrier Initiator prion hypothesis
}

\author{
Kavitha Reddy ${ }^{1}$ \\ ${ }^{1}$ Affiliation not available
}

May 5, 2020

Beyond the protein-only hypothesis: the Lipid-Carrier Initiator prion hypothesis

Kavitha Reddy, M.D. ${ }^{1}$

${ }^{1}$ Boston, Massachusetts

\section{Introduction}

Abnormally-folded proteins occur in several diseases that affect humans and animals, known as prion diseases. Prion diseases include sporadic and variant Creutzfeldt-Jakob disease (CJD), Kuru, bovine spongiform encephalopathy (BSE, or mad cow disease), scrapie, and chronic wasting disease (CWD). Known prion diseases result in progressive neurodegenerative disease, leading to early death. Alzheimer's disease (AD), Parkinson's disease (PD), and Amyotrophic lateral sclerosis (ALS) are also considered by many as under the umbrella of prion disease, because of the observed parallels between members of the entire group (1). Accordingly, an understanding of traditional transmissible spongiform encephalopathies (TSE) is likely to contribute significantly to an improved understanding of the more frequently seen Alzheimer's disease, Parkinson's disease, and Amyotrophic lateral sclerosis (ALS), which in total affect millions of individuals worldwide. Similarly, an improvement in the understanding of the pathogenesis of AD, PD, or ALS may provide significant insight into the entire group of prion diseases. Unfortunately the mechanism of protein misfolding in both human and animal diseases has remained poorly understood, and there has been little progress to date in improving outcomes (1).

The protein-only hypothesis has provided for many valuable steps in understanding, although there remains a further need for a more complete model of the mechanism of protein misfolding. Novel theories, such as that described herein, may provide alternative and adjunctive explanations that can improve our understanding of all prion diseases. The mineral-lipid hypothesis has been described in a previous manuscript and represents a novel theory to explain the formation and propagation of prions (1). The aim of this shorter manuscript is to expand this hypothesis to consider whether it may represent a special case, or particular example of, a more encompassing "Lipid-Carrier Initiator" prion theory, which is described herein.

\section{The Lipid-Carrier Initiator Prion Hypothesis}

In the Lipid-carrier initiator hypothesis, one may consider that prions form by occurrence of the following steps.

1. A charged, curved particle is present. Under natural conditions, often this may be a negatively charged round lipid particle, such as a lipid vesicle. We will term this the primary initiator.

A curved, charged lipid particle may be created by the presence of an anion and lipids. For example, ribonucleic acid (RNA), or the aluminosilicate mineral montmorillonite (Mte), when combined with lipids, creates lipid vesicles. Other curved lipid particles are naturally present inside and outside of cells, serving a variety of functions. 
2. A secondary portion of the initiator unit (we will use the term secondary initiator) may be present and bonds to or associates with the target protein or the lipids in its vicinity, and functions as an agent promoting conformational change.

For example, the secondary initiator may promote the final conformational change by disrupting and forming hydrogen bonds, hydrophobic or hydrophilic effects, increasing the disorder of the target protein structure, influencing local charge interactions, increasing beta-sheet tendency or increasing the stability of an alternate folding conformation, and/or functioning as an aggregant.

The target protein refers to the protein that becomes misfolded and is commonly associated with the disease state, e.g. Prion protein (PrP), A-beta, Alpha-synuclein, or superoxide dismutase 1 (SOD1).

3. The secondary initiator may be the assembler, carrier, or attractor of the primary initiator round lipid particle. In fact, potential examples of this seem to provide the most elegant and efficient natural design.

For example, RNA or mineral (Mte) might act as secondary initiators, as they can aggregate or bind prion protein, while also associating with lipid vesicles. Misfolded or mobile target proteins themselves may also fit these characteristics; for example prion protein $\mathrm{PrP}^{\mathrm{Sc}}$ associates with lipid and also binds or interact with further PrP.

In this hypothesis, amphiphiles may also be considered as candidates for secondary initiators. For example, ApoE4, a prominent risk factor for Alzheimer's disease, might act as a secondary initiator. ApoE4 carries a lipid particle at one portion of the molecule. ApoE4 also has a portion that exhibits poor stability and contains amino acids associated with tendencies toward beta-sheet formation (2). According to this Lipid-carrier initiator hypothesis, this portion of the apoE4 molecule could be considered as promoting conformational change and beta-tendency in the target protein upon interaction with the target protein. The combined effects of the primary initiator lipid particle that is carried by ApoE4 and the secondary initiator which has the capacity to further interact with and change the shape of the target protein, could produce the conformational change in the target protein. By this process, a misfolded protein may be created.

4. The protein misfolding process described may be viewed as analogous to the initiation step of a polymerization process, converting an original alpha-helical monomer into a polymerizable (activated) beta-sheet form. As the process continues forward, further activations result in polymer propagation, observed as fibril or plaque formation.

\section{Discussion}

When considering this hypothesis, one may also consider out of an abundance of caution as to whether injection, ingestion, or other exposure to substances containing component(s) of the above described lipidcarrier initiator unit could, in a percentage of those exposed, lead to initiation of protein misfolding or to the spread and propagation of existing misfolded protein presently localized at the site of exposure, and ultimately lead to the development of clinical prion disease. This outcome might be considered of theoretical concern even if only one of the above components of the lipid-carrier initiator prion unit is delivered as a result of exogenous, environmental, or iatrogenic intervention, because the others may be present in vivo already due to natural conditions in the exposed tissue. In addition, possible protein-misfolding related effects may be considered when assessing and optimizing safety of the many liposomal and nanoparticle products that are being developed in modern science.

\section{Western Pacific ALS/P-D: Exploration of potential Cycad plant toxicity}

Clusters of disease showing amyotrophic lateral sclerosis, parkinsonism-dementia, or an overlap of symptoms of both diseases (Western Pacific ALS/P-D) affected local individuals during a recent limited historical period on the island of Guam, the Kii pensisula of Japan, and a portion of New Guinea (3). In Guam, the local disease is commonly called lytico-bodig. The preponderance of data collected to date strongly suggests an environmental cause for Western Pacific ALS/P-D. Cycad plant exposure has been identified as common 
to the three geographic sites (3). In Guam, individuals reported an increase in consumption of Cycad flour during the lifetimes of those affected with the disease. Cycad seeds have been used by local individuals in the affected area of New Guinea for topical wound care as a poultice, including for deep wounds. Cycad has also been available in some affected regions as an oral medication. Cycad exposure has been considered by many to be the most likely cause of Western Pacific ALS/P-D. A number of other theories have also been explored. While intense interest remains in understanding Western ALS/P-D, the cause has not been confirmed to date.

Because raw cycad consumption by humans and animals is well-documented to cause a potent neurotoxic effect, called neurocycadism, with acute poisoning signs and often long-term non-progressive neurologic deficits, it is accepted that cycads contain neurotoxin(s) (3). To that end, neurotoxic chemicals produced by the cycad plant such as cycasin (methylazoxymethanol (MAM)) have been studied as possible causes of the progressive, slower disease of Western Pacific ALS/P-D. However, a clear link of cycads to ALS/P-D has not yet been established.

It may be useful to consider additional hypotheses as to how cycad exposure could cause a progressive neurologic disease resembling ALS/P-D. Here we will for interest and exploration reconsider the cycad hypothesis in the light of the Lipid-carrier initiator hypothesis. One may consider the following potential factors in cycads that could promote prion formation.

Cycad seeds contain lipids, such as plant seed lipid-bodies, that are often in the form of vesicles or spherules. (4). These may be long-lasting and are stabilized by proteins, including caleosin in cycads. These could be a potential source of initiators. One should also note that clay was sometimes used in the process of cycad flour preparation, and the mineral-lipid hypothesis should not be entirely excluded. For example, there is a description that those preparing cycad seeds would often coat their hands in clay during the process. Clay minerals can bind and hold neurotoxins, and can combine with lipids to make vesicles. A number of plants produce antimicrobial peptides (AMPs), to aid in defense of the plant against infection. Cycad plants produce three known antimicrobial peptides, named Cy-AMP1, Cy-AMP2, and Cy-AMP3 (5). Cy-AMP3 may be related to lipid transfer. The majority of Cy-AMPs have significant cysteine, proline and glycine content (5). I note with relationship to the lipid-carrier initiator hypothesis that these amino acids are associated with beta-sheet tendencies, making the Cy-AMPs possible secondary initiators, and that AMPs are amphiphilic, so one might consider that they could interact with target protein and associate with primary initiator lipid particles. AMPs have several membrane and lipid-related effects, including the capacity to disrupt and alter membranes, to form smaller particles from the lipid bilayer, to form non-lamellar shapes from membrane lipids, to associate closely with lipid particles, and the ability to bind to proteins (6). Therefore, if one applies the lipid-carrier initiator hypothesis, Cy-AMPs could provide a source of lipid vesicles by disrupting membrane, if the needed lipid vesicle source is not already provided by seed lipid bodies, and might carry or associate closely with lipid particles or affect lipids near the target protein. CyAMPs could act as secondary initiators by also binding to or associating with target protein. Alternatively, the non-specific effect of membrane disruption, could itself promote mixing of lipids with components such as cellular RNA, mixing of lipids with target proteins, mobilization of pre-existing misfolded proteins, or creation of misfolded proteins by the process of membrane disruption and shape change. These factors could promote prion disease in some individuals exposed to Cycads.

The mineral-lipid and the lipid-carrier initiator hypotheses allow us to expand beyond the protein-only model of the pathogenesis of prion and protein-misfolding diseases. These hypotheses provide a potential explanation for a broad array of seemingly disparate factors across multiple prion diseases. There is value in continuing to encourage discussion and in encouraging theories that can explain many aspects of prion diseases in a comprehensive model. Affected individuals and families are served by our willingness to think broadly and creatively about possible models and solutions. With the continued efforts of many, these diseases will one day be understood well and effective interventions will become available.

\section{References}


1. K. Reddy, Beyond the protein-only hypothesis: A novel mineral-lipid hypothesis in prion and proteinmisfolding disease. Peer J Preprints (2017). https://doi.org/10.7287/peerj.preprints.2982v1

2. N. Zhong, K.H. Weisgraber, Understanding the association of apolipoprotein E4 with Alzheimer disease: clues from its structure. J Biological Chemistry, 284:10, 6027-6031 (2009).

3. G. E. Kisby, P. S. Spencer, Is neurodegenerative disease a long-latency response to early-life genotoxin exposure? Int J Environ Res Public Health, 8, 3889-3921 (2011).

4. K.D. Chapman, J.M. Dyer, R. T. Mullen, Biogenesis and function of lipid droplets in plants. J Lipid Research, 53, 215-226 (2012).

5. S. Yokoyama, K. Kato, A. Koba, Y. Minami, K. Watanabe, F. Yagi, Purification, characterization, and sequencing of antimicrobial peptides Cy-AMP1, Cy-AMP2, and Cy-AMP3, from the Cycad (Cycas revoluta) seeds. Peptides, 29, 2110-2117 (2008).

6. A. A. Bahar, D. Ren, Antimicrobial peptides. Pharmaceuticals, 6, 1543-1575 (2013). 